\title{
Homogeneous Anisotropic Bianchi Type IX Cosmological Model For Perfect Fluid Distribution With Electromagnetic Field
}

\author{
Atul Tyagi ${ }^{1, *}$, Dhirendra Chhajed ${ }^{2}$ \\ ${ }^{1}$ Department of Mathematics \& Statistics, University College of Science, Mohan Lal Sukhadia University, Udaipur, 313001, India \\ ${ }^{2}$ Department of Mathematics, Sir Padampat Singhania University, Udaipur, 313601, India
}

\begin{abstract}
We have investigated homogeneous anisotropic Bianchi type IX cosmological model for perfect fluid distribution with electro-magnetic field. The magnetic field is due to an electric current produced along x axis thus $F_{23}$ is the only non-vanishing component of electromagnetic field tensor $F_{i j}$. To get a determinate model, we have assumed the condition $a=b^{n}$ between metric potentials, where $n$ is constant. The various physical and geometrical aspects of the model viz pressure, density, shear, expansion factor, electromagnetic field tensor and conformal curvature are also discussed.
\end{abstract}

Keywords Bianchi type IX, Cosmological Model, Anisotropic, Perfect Fluid, Electromagnetic Field

\section{Introduction}

Many relativists have taken keen interest in studying Bianchi IX universe because familiar solution like Robertson Walker universe, the de-sitter universe. The Taub-NUT solutions etc. are of Bianchi IX space times. Bianchi IX cosmological models include closed FRW models. These models allow not only expansion but also rotation and shear and in general are anisotropic. In recent years cosmological models with rotation and expansion have been constructed by many research workers viz. Novello and Reboucas[1] , Reboucas and Lima[2,3] , Rosquist[4], Bradley and Svienstins[5], Svienstins[6], Patel and Yadava[7]. Homogeneous and anisotropic cosmological models have been widely studied in the frame work of general relativity in the search of realistic picture of the universe in its early stages because they explain a number of observed phenomena quite satisfactorily.

The observational relations for discrete sources in certain homogeneous anisotropic model have been derived by Saunders[8, 9], Tomita[10], Mac-Callum and Ellis[11].

Ftaclas \& Cohen[12] have investigated LRS (Locally Rotationally Symmetric) Bianchi type V universes containing stiff matter with electromagnetic field. Lorentz[13,14 ] investigated Bianchi type cosmologies in presence of magnetic field. Assad and Soares[15] have investigated a class of exact cosmological solution of Einstein's - Maxwell

* Corresponding author:

tyagi.atul10@gmail.com (Atul Tyagi)

Published online at http://journal.sapub.org/ajms

Copyright (C) 2012 Scientific \& Academic Publishing. All Rights Reserved equations, which are anisotropic and spatially homogeneous. Bianchi VIII and IX locally rotationally symmetric model.

The dynamical effects of spatially homogeneous electromagnetic field on anisotropic Bianchi type IX models are studied by Waller[16]. Brill[17] studied Einstein's - Maxwell equations in a homogeneous and non-isotropic universe.

Bali and Dave[18] have investigated Bianchi type IX string cosmological model in General Relativity. Bali and Kumawat[19] have investigated Bianchi type IX stiff fluid tilted cosmological model with bulk viscosity. Tyagi and Sharma[20] investigated Bianchi IX string cosmological model for perfect fluid distribution in General Relativity.

In this paper, we have investigated homogeneous anisotropic Bianchi type IX cosmological model of perfect fluid distribution with electro-magnetic field. We have assumed that $F_{23}$ is the only non-vanishing component of electromagnetic field tensor $F_{i j}$.

To get a determinate model, we have assumed the condition $a=b^{n}$ between metric potentials, where $n$ is constant. The various physical and geometrical aspects of the model are also discussed.

\section{Formations of Field Equations}

We consider homogeneous anisotropic Bianchi type IX metric in the form

$$
\begin{aligned}
& d s^{2}=-d t^{2}+a^{2}(t) d x^{2}+b^{2}(t) d y^{2}+ \\
& \left(b^{2} \sin ^{2} y+a^{2} \cos ^{2} y\right) d z^{2}-2 a^{2} \cos y d x d z
\end{aligned}
$$

Where metric potentials $a \& b$ are functions of 't' alone. The energy momentum tensor is taken in the form

$$
\mathrm{T}^{j}{ }_{i}=(\varepsilon+p) v_{i} v^{j}+p g^{j}{ }_{i}+E^{j}{ }_{i}
$$


$\mathrm{p}$ being the pressure, $\varepsilon$ the density.

The co-ordinates are considered to be comoving so that $v^{1}=0=v^{2}=v^{3} \& v^{4}=1$ for the line element (1)

The electromagnetic field $E^{j}{ }_{i}$ is defined by

$$
\mathrm{E}_{\mathrm{i}}^{\mathrm{j}}=\frac{1}{4 \pi}\left[-F_{i l} F^{j l}+\frac{1}{4} g^{j}{ }_{i} F_{l m} F^{l m}\right]
$$

Maxwell's equation

$$
\frac{\partial}{\partial x^{j}}\left(F^{i j} \sqrt{-g}\right)=0
$$

Equation (4) leads to

$$
F_{23}=H \sin y
$$

The Einstein's field equation

$\mathrm{R}^{j}{ }_{i}-\frac{R}{2} g^{j}{ }_{i}=-8 \pi T^{j}{ }_{i}$ for the line element (1) are given by

$$
\begin{array}{r}
2 \frac{b_{44}}{b}+\frac{b_{4}^{2}}{b^{2}}+\frac{1}{b^{2}}-\frac{3 a^{2}}{4 b^{4}}+\Lambda=-8 \pi\left(p+\frac{H^{2}}{8 \pi b^{4}}\right) \\
\frac{a_{44}}{a}+\frac{b_{44}}{b}+\frac{a_{4} b_{4}}{a b}+\frac{a^{2}}{4 b^{4}}+\Lambda=-8 \pi\left(p-\frac{H^{2}}{8 \pi b^{4}}\right) \\
2 \frac{a_{4} b_{4}}{a b}+\frac{b_{4}^{2}}{b^{2}}+\frac{1}{b^{2}}-\frac{a^{2}}{4 b^{4}}+\Lambda=-8 \pi\left(-\varepsilon+\frac{H^{2}}{8 \pi b^{4}}\right)
\end{array}
$$

\section{Solutions of the Field Equations}

Equations (6) - (8) are three equations in four unknown $a$, $b, \varepsilon$ and $p$.

To get a determinate solution, we assume the condition between metric potentials

$$
a=b^{n}
$$

Equation (6) and (7) together lead to

$$
\frac{a_{44}}{a}-\frac{b_{44}}{b}+\frac{a_{4} b_{4}}{a b}-\frac{b_{4}^{2}}{b^{2}}-\frac{1}{b^{2}}+\frac{a^{2}}{b^{4}}=\frac{K}{b^{4}}
$$

Where $K=2 H^{2}$

Using the condition (9) in (10), we get

$$
b b_{44}+(n+1) b_{4}^{2}=\frac{1}{n-1}\left[1+\frac{K}{b^{2}}-\frac{1}{b^{2-2 n}}\right]
$$

Putting $b_{4}=f(b)$ in equation (11), we get

$$
b f f^{\prime}+(n+1) f^{2}=\frac{1}{n-1}\left[1+\frac{K}{b^{2}}-\frac{1}{b^{2-2 n}}\right]
$$

Equation (12) leads to

$$
\frac{d f^{2}}{d b}+\frac{2}{b}(n+1) f^{2}=\frac{2}{n-1}\left[\frac{1}{b}+\frac{K}{b^{3}}-\frac{1}{b^{3-2 n}}\right]
$$

Equation (13) leads

$$
f^{2}=\frac{1}{n-1}\left[\frac{1}{n+1}+\frac{K}{n b^{2}}-\frac{1}{2 n b^{2-2 n}}\right]+\frac{L}{b^{2 n+2}}
$$

Where $\mathrm{L}$ is constant of integration. (14)

Equation (14) leads to

$$
\int \frac{\mathrm{db}}{\sqrt{\frac{1}{(\mathrm{n}-1)}\left[\frac{1}{\mathrm{n}+1}+\frac{\mathrm{K}}{\mathrm{nb} b^{2}}-\frac{1}{2 n b^{2-2 n}}\right]+\frac{\mathrm{L}}{\mathrm{b}^{2 n+2}}}}=\mathrm{t}+\mathrm{M}
$$

Where $\mathrm{M}$ is constant of integration, value of $\mathrm{b}$ can be determined from equation

The metric (1) reduces to form

$$
\begin{aligned}
d s^{2}= & \frac{1}{n-1}\left[\frac{1}{n+1}+\frac{K}{n T^{2}}-\frac{1}{2 n T^{2-2 n}}\right]+\frac{L}{T^{2 n+2}} \\
& +T^{2} d Y^{2}+\left(T^{2} \sin ^{2} Y+T^{2 n} \cos ^{2} Y\right) d Z^{2} \\
& -2 T^{2 n} \cos Y d X d Z
\end{aligned}
$$

Where $b=T, x=X, y=Y, z=Z$

\section{Some physical and Geometrical Features}

Pressure and density for the model (16) are given by

$$
\begin{aligned}
8 \pi p & =\frac{n^{2}}{\left(1-n^{2}\right) T^{2}}+\frac{K\left(n^{2}-n-2\right)}{2 n(1-n) T^{4}}+\frac{(3 n-2)(n+1)}{4 n(n-1) T^{4-2 n}} \\
& +\frac{(2 n+1) L}{T^{2 n+4}}-\Lambda \\
8 \pi \varepsilon= & \frac{n(n+2)}{\left(n^{2}-1\right) T^{2}}+\frac{(n+2)(n+1) K}{2 n(n-1) T^{4}}+\frac{(n+1)(n+2)}{4 n(1-n) T^{4-2 n}} \\
& +\frac{(2 n+1) L}{T^{2 n+4}}+\Lambda
\end{aligned}
$$

The condition $\varepsilon>0$ leads to

$$
\frac{n(n+2)}{\left(n^{2}-1\right) T^{2}}+\frac{(n+2)(n+1) K}{2 n(n-1) T^{4}}+\frac{(n+1)(n+2)}{4 n(1-n) T^{4-2 n}}+\frac{(2 n+1) L}{T^{2 n+4}}+\Lambda>0
$$

Condition $\varepsilon>p$ leads to

$$
\frac{2 n}{(n-1) T^{2}}+\frac{K(n+1)}{(n-1) T^{4}}+\frac{(n+1)}{(1-n) T^{4-2 n}}+2 \Lambda>0
$$

Which is condition on $\Lambda$

The charge current vector $J^{i}$ is given by

$$
\mathrm{J}^{\mathrm{i}}=\left(\frac{1}{\bar{\mu}} \mathrm{F}^{\mathrm{ij}}\right)_{; \mathrm{j}}
$$

This turns out to be zero.

The scalar of expansion $\theta$ calculated for the flow vector $\mathrm{v}^{i}$ is given by

$$
\theta=(n+2) \sqrt{\frac{1}{n-1}\left[\frac{1}{T^{2}(n+1)}+\frac{K}{n T^{4}}-\frac{1}{2 n T^{4-2 n}}\right]+\frac{L}{T^{2 n+4}}}
$$

The rotation $\omega$ is identically zero. The non-vanishing components of Shear $\sigma_{i j}$ are given by

$$
\begin{aligned}
& \sigma_{11}=\frac{2}{3} T^{2 n}(n-1) . \\
& \sqrt{\frac{1}{n-1}\left[\frac{1}{T^{2}(n+1)}+\frac{K}{n T^{4}}-\frac{1}{2 n T^{4-2 n}}\right]+\frac{L}{T^{2 n+4}}} \\
& \sigma_{22}=\frac{1}{3} \mathrm{~T}^{2}(1-n) \sqrt{\frac{1}{\mathrm{n}-1}\left[\frac{1}{\mathrm{~T}^{2}(\mathrm{n}+1)}+\frac{\mathrm{K}}{\mathrm{nT}^{4}}-\frac{1}{2 \mathrm{nT} \mathrm{T}^{4-2 \mathrm{n}}}\right]+\frac{\mathrm{L}}{\mathrm{T}^{2 \mathrm{n}+4}}} \\
& \sigma_{33}=\frac{1}{3}\left(-T^{2} \sin ^{2} Y+2 T^{2 n} \cos ^{2} Y\right)(n-1) \\
& \sqrt{\frac{1}{n-1}\left[\frac{1}{T^{2}(n+1)}+\frac{K}{n T^{4}}-\frac{1}{2 n T^{4-2 n}}\right]+\frac{L}{T^{2 n+4}}}
\end{aligned}
$$




$$
\begin{aligned}
\sigma_{13}=\sigma_{31}= & \frac{-2 T^{2 n}}{3} \cos Y(n-1) \\
& \sqrt{\frac{1}{n-1}\left[\frac{1}{T^{2}(n+1)}+\frac{K}{n T^{4}}-\frac{1}{2 n T^{4-2 n}}\right]+\frac{L}{T^{2 n+4}}}
\end{aligned}
$$

Hence

$$
\sigma^{2}=\frac{1}{3}(n-1)^{2}\left[\frac{1}{(n-1)}\left(\frac{1}{T^{2}(n+1)}+\frac{K}{n T^{4}}-\frac{1}{2 n T^{4-2 n}}\right)+\frac{L}{T^{2 n+4}}\right]
$$

The non-vanishing components of conformal curvature tensor are given by

$$
\begin{aligned}
C_{12}^{12}= & \frac{1}{6}\left(1+T^{2 n-2} \frac{\cos ^{2} Y}{\sin ^{2} Y}\right) \\
& {\left[\left(\frac{2-n}{n}\right) \frac{K}{T^{4}}+\frac{2 n-1}{n T^{4-2 n}}-\frac{2 n}{(n+1) T^{2}}+\frac{2(n-1) L}{T^{2 n+4}}\right] } \\
C_{13}^{13}= & \frac{1}{6}\left(1+T^{2 n-2} \frac{\cos ^{2} Y}{\sin ^{2} Y}\right) \\
& {\left[\left(\frac{2-n}{n}\right) \frac{K}{T^{4}}+\frac{2 n-1}{n T^{4-2 n}}-\frac{2 n}{(n+1) T^{2}}+\frac{2(n-1) L}{T^{2 n+4}}\right] } \\
C_{14}^{14}= & -\frac{1}{3}\left(1+T^{2 n-2} \frac{\cos ^{2} Y}{\sin ^{2} Y}\right) \\
& {\left[\left(\frac{2-n}{n}\right) \frac{K}{T^{4}}+\frac{2 n-1}{n T^{4-2 n}}-\frac{2 n}{(n+1) T^{2}}+\frac{2(n-1) L}{T^{2 n+4}}\right] }
\end{aligned}
$$

\section{Conclusions}

The expansion in the model decreases as time increases. The expansion stop at $\mathrm{T}=\infty$ and also when $n=-2$. since $\lim _{T \rightarrow \infty} \frac{\sigma}{\theta} \neq 0$, therefore, the model doesn't approach isotropy for large value of $\mathrm{T}$. However when $n=1$ the model is isotropize. When $K \rightarrow 0$ then $\theta \rightarrow(n+2) \sqrt{\frac{1}{n-1}\left[\frac{1}{T^{2}(n+1)}-\frac{1}{2 n T^{4-2 n}}\right]+\frac{L}{T^{2 n+4}}}$ which show that the expansion in the model tend to zero for large value of $\mathrm{T}$ in the absence of magnetic field. The space-time is petrov type-D.

In general, the model represent expanding, shearing and non- rotating universe.

\section{REFERENCES}

[1] Novello, M \& Reboucas, M.J. (1978) Astrophys, J 225, 719
[2] Reboucas, M.J. and Lima, J.A.S. (1982) J. Maths. Phys. 23, 1547

[3] Reboucas, M.J. and Lima, J.A.S. (1982) J. Maths. Phys. 29 , 2699

[4] Rosquist, K (1983) phys .97 A, 145

[5] Bradely M. and Svienstins, E.(1983) Gen. Relativity Gravitation 16,1119

[6] Svienstins, E.(1985) ) Gen. Relativity Gravitation. 17, 54

[7] Patel, L.K. and Yadav S.P.(1987) Indian J. of pure \& applied Math. 18(9), 840-845

[8] P.T. Saunder, Observations in homogeneous model universes. Mon. Not. R.. Astr. Soc. 141, (1968), 427-437.

[9] P.T. Saunder, Observations in some simple cosmological Models with shear. (1969)Mon. Not. R. astro. Soc. 142, 213-227.

[10] K. Tomita, Theoretical relations among observable quantities in an anisotropic \& Homogeneous Universe. Progress. theoretical phys. (1968) 40, 2, 264-276

[11] M.A.H. Mac Callum \& G.F.R. Ellis, A class of homogeneous cosmological models II: observations (1970) Comm. Math. Phys. 19, 1, 31-64.

[12] C. Ftaclas, \& J.M. Cohen, LRS(Locally Rotationally Symmetric) Bianchi type $\mathrm{V}$ universes containing stiff matter with electromagnetic field, (1978)Phys. Rev D 184373.

[13] D. Lorentz, An exact Bianchi type V tilted cosmological Model with matter \& an Electromagnetic field,(1981) G.R.G. $13,8,795-805$.

[14] D. Lorentz, Exact Bianchi type $V I_{0}$ cosmological models with matter \& and electromagnetic field,(1982) Atrophy's. \& Space Science. 85, 1-2, 69-74.

[15] M.J.D. Assad and I. Damio Soares , Anisotropic Bianchi types VIII \& IX locally rotationally symmetric cosmologies, Phys. Rev D.28, 8, (1983), 1858-1865.

[16] S.M.Waller, Bianchi type IX electromagnetic universes, (1984)P hys. Rev. D. 29, 2, 176-185.

[17] D.R.Brill, Electromagnetic fields in a Homogeneous, Non isotropic universe. (1964) Phys, Rev. 133, 3B B845-B848.

[18] Bali, R \& Dave S. Bianchi type IX string cosmological model in General Relativity. Pramana J. of physics,(2001), 56,4

[19] Bali, R \& Kumawat P Bianchi type IX stiff fluid tilted cosmological model with bulk viscosity(201 EJTP 7, No 24 , 383-394

[20] Tyagi,A \& Sharma K Bianchi IX string cosmological model for perfect fluid distribution in General Relativity(2010) CHIN.PHYS.LETT. Vol 27, No 7079801 